\title{
Longitudinal associations of affective symptoms with mid-life cognitive function: evidence from a British birth cohort
}

Amber John, Sarah-Naomi James, Urvisha Patel, Jennifer Rusted, Marcus Richards and Darya Gaysina

\section{Background}

Affective disorders are associated with poorer cognition in older adults; however, whether this association can already be observed in mid-life remains unclear.

\section{Aims}

To investigate the effects of affective symptoms over a period of 30 years on mid-life cognitive function. First, we explored whether timing (sensitive period) or persistence (accumulation) of affective symptoms predicted cognitive function. Second, we tested how different longitudinal trajectories of affective symptoms were associated with cognitive function.

\section{Method}

The study used data from the National Child Development Study. Memory, verbal fluency, information processing speed and accuracy were measured at age 50 . Affective symptoms were measured at ages $23,33,42$ and 50 and used to derive longitudinal trajectories. A structured modelling approach compared a set of nested models in order to test accumulation versus sensitive period hypotheses. Linear regressions and structural equation modelling were used to test for longitudinal associations of affective symptoms with cognitive function.

\section{Results}

Accumulation of affective symptoms was found to be the best fit for the data, with persistent affective symptoms being associated with poorer immediate memory $(b=-0.07$, s.e. $=$ $0.03, P=0.01)$, delayed memory $(b=-0.13$, s.e. $=0.04, P<0.001)$ and information processing accuracy $(b=0.18$, s.e. $=0.08, P=$ $0.03)$, but not with information processing speed $(b=3.15$, s.e. $=$ $1.89, P=0.10$ ). Longitudinal trajectories of repeated affective symptoms were associated with poorer memory, verbal fluency and information processing accuracy.

\section{Conclusions}

Persistent affective symptoms can affect cognitive function in mid-life. Effective management of affective disorders to prevent recurrence may reduce risk of poor cognitive outcomes and promote healthy cognitive ageing.

\section{Declaration of interest}

None.

\section{Keywords}

Depressive disorders; epidemiology; anxiety disorders; ageing; cognition; dementia; Iongitudinal.

\section{Copyright and usage}

(c) The Royal College of Psychiatrists 2019
Affective disorders, such as depression and anxiety, have been associated with impaired cognition, dementia and accelerated cognitive decline in late adulthood. ${ }^{1-3}$ However, there are significant gaps in our understanding of this association. The majority of studies have been conducted in older adults, usually over the age of 60 and have only considered this association in the context of short follow-up periods ( $<10$ years). ${ }^{4}$ Therefore, it is unclear how affective symptoms are associated with cognitive function over time. There is some evidence that risk of dementia increases with the number of affective episodes experienced over the life course; whereby rate of dementia is estimated to increase by around $13 \%$ with each additional depressive episode. ${ }^{5}$ Additionally, evidence shows that individuals with persistent depressive symptoms may be at greater risk for subsequent cognitive deficits than individuals with fewer episodes. ${ }^{4}$ Another line of evidence suggests that timing, rather than frequency, of affective symptoms can be an important predictor of cognitive outcomes. For example, depressive symptoms in older age only were shown to be associated with increased risk of dementia. ${ }^{6}$ However, there is limited inconsistent evidence for associations between affective disorders and cognitive function before old age in two studies using data from the Medical Research Council (MRC) National Survey of Health and Development. The first study ${ }^{7}$ found no associations of affective symptoms measured between ages 13 to 53 with objectively measured cognitive outcomes at age 60-64, whereas the most recent study reported that persistent case-level affective symptoms were associated with poorer cognitive state, verbal memory and letter search speed and accuracy at age $69 .{ }^{8}$

In this current study we tested for associations of affective symptoms across adulthood (ages 23, 33, 42 and 50) with aspects of cognitive function at age 50. Specifically, two complementary advanced statistical approaches were employed. First, a structured modelling approach comparing a set of nested models was used to investigate whether accumulation or timing of case-level affective symptoms can predict mid-life cognition. Second, a latent growth mixture modelling approach was employed to derive longitudinal trajectories of affective symptoms and to examine how different longitudinal trajectories of affective symptoms were associated with mid-life cognition.

\section{Method}

\section{Participants}

Participants were from the National Child Development Study (NCDS), a population representative sample of 18558 people born during 1 week of 1958 in England, Scotland and Wales (i.e. British 1958 birth cohort). This sample comprised $98.2 \%$ of total births that week. Data were subsequently collected from participants at ages $7,11,16,23,33,42,46$ and 50 years. Further information about the study sample, respondent profiles and data collection methods are described in depth by Power \& Elliott. ${ }^{9}$ Data are available from the UK Data Service. ${ }^{10-14}$ Cohort members gave written 
informed consent to participate. Ethical approval for the data collection in 2008 was provided by London Medical Research and Ethics Committee (REC reference: 08/H0718/29). Ethical approval for the present study was also provided by the University of Sussex (Reference number: ER/AJ316/1).

Detailed information about the sample analysed in the present study is presented in supplementary File 1, supplementary Fig. 1 and supplementary Table 1 available at https://doi.org/10.1192/ bjp.2019.24. There were 9790 people who participated in the 2008 data collection (at age 50). A total of 9385 people in this sample completed the cognitive tests at this time point (95.9\%). Of the 9385 participants who completed the cognitive tests at age 50, 4625 had complete information for all predictors and covariates (49.3\% of those with cognitive measures).

\section{Measures}

\section{Cognitive function}

Cognitive measures collected at age 50 were verbal memory, verbal fluency, information processing speed and accuracy. ${ }^{15}$ Memory was assessed using a word-recall test with immediate and delayed components. Verbal fluency was assessed using an animal naming task, in which participants named as many animals as possible in a $1 \mathrm{~min}$ period. Information processing was assessed using a letter cancellation task. In this task, cohort members crossed as many target letters as possible from a grid of letters in a $1 \mathrm{~min}$ period. The results of this task were split into two scores. The total number of letters scanned represented information processing speed, and the number of target letters missed up to the final letter searched represented information processing accuracy. Accuracy was negatively scored, so higher scores represent poorer performance.

\section{Affective symptoms}

Affective symptoms were assessed using the Malaise Inventory Scale, administered at ages $23,33,42$, and $50 .{ }^{16-18}$ The items in the malaise inventory scale are available online at: https:// www.ukdataservice.ac.uk/teaching-resources/malaise/questions.asp. This is a measure of psychological distress and comprises 24 selfcompletion items, which are combined to assess levels of emotional disturbance and associated somatic symptoms. The total number of questions answered 'yes' was summed, creating a sum score of affective symptoms out of 24 . This score was dichotomised using a standard cut-off threshold score of 7 out of 24 to represent 'case-level' affective symptoms, indicating clinically relevant affective symptoms. ${ }^{16-18}$ At age 50, a short form of the Malaise Inventory Scale was administered, comprising nine items. For this time point, a recommended cut-off score of three was used to categorise caselevel affective symptoms. ${ }^{19}$

As the short form of the Malaise Inventory Scale was used at age 50 , the nine items from this short form were extracted from the longer forms used at ages 23,33 , and 42 , in order to make malaise scores over time more comparable to be used for modelling longitudinal trajectories.

\section{Covariates}

Based on previous research, the following factors were included as potential confounders: gender, childhood cognition, ${ }^{20}$ childhood emotional adjustment, ${ }^{21}$ childhood socio-economic position, ${ }^{22}$ adult socio-economic position ${ }^{23}$ and education. ${ }^{24}$ Childhood cognition was assessed at age 11, using a general ability test administered at the child's school. Childhood emotional adjustment was assessed at age 11 using the Bristol Social Adjustment Guides. ${ }^{25}$ This questionnaire was completed by teachers and is designed to assess behaviour that may be indicative of maladjustment and emotional disturbance.
A measure of household socio-economic position at age 11 was derived using guidelines from the Centre for Longitudinal Studies, ${ }^{26}$ based on measures of father's occupation, mother's occupation and household tenure. Highest educational attainment was derived by combining education data from 1991, 2000, 2004 and 2008 to ascertain the highest academic qualification the cohort member had achieved by age 50. Adult socio-economic position was based on occupation, with three categories (working, intermediate and middle class). Additional information about these covariates are included in supplementary File 2.

\section{Analytical procedure}

First, a structured modelling approach was used to compare a set of nested models corresponding to accumulation and sensitive period hypotheses to a saturated model including all main effects and all possible interactions. ${ }^{8,27,28}$ The sensitive period model included three measures indicating whether an individual experienced caselevel affective symptoms at three time windows across the life course: early adulthood (age 23); middle adulthood (ages 33 and/or 42); and mid-life (age 50). The accumulation model included a measure of the number of time points (of the three windows of time described above) in which each individual experienced case-level affective symptoms. The saturated model was also compared with a 'null' model that assumed no effect of affective symptoms on midlife cognition. Partial F-tests were used to compare each hypothesis to the fully saturated model. Where multiple $P$-values were $>0.05$, the model with the highest $P$-value and lowest $F$-statistic were selected as the best fit for the data. This analysis was conducted in Stata (V14.2).

Four variables with case-level affective symptoms were summed up to create a variable for accumulation of adult affective symptoms (ranging from 0 to 4). Linear regression models were fitted to test for associations of accumulation of adult affective symptoms and cognitive function at age 50 . For these analyses, four models were fitted: unadjusted (model 1); adjusted for gender only (model 2); additionally, adjusted for child cognition, emotional disturbance and socio-economic position (model 3); and additionally, adjusted for highest educational attainment, and adult socio-economic position (model 4). This analysis was conducted in R (V3.5.1).

To account for missing data, multiple imputation analysis was conducted on the sample with cognitive data. All analyses described above were re-run using imputed variables for the key predictors. Multiple imputation was conducted in $\mathrm{R}$ using the MICE package. ${ }^{29,30}$ Twenty imputations were conducted using data across seven sweeps over the life course. This multiple imputation approach includes a large number of covariates and auxiliary variables in the models, which maximises the plausibility of the missing at random assumption, and limits possibility of missing not at random data. ${ }^{31}$ These multiple imputation techniques have been used extensively to address missing data in the NCDS. ${ }^{32-34}$ Technical details of the multiple imputation process are reported in supplementary File 3.

Second, a confirmatory factor analysis was conducted to generate latent factor scores of affective symptoms at each time point (see supplementary Table 2 for details about model fit). Linear mixed models were then used to examine affective symptom trajectories over time, using factor scores from each time point. Linear and quadratic models were fitted and compared and the model with the best fit according to the Akaike information criterion (AIC) and Bayesian information criterion (BIC) was selected for subsequent analyses. Next, growth mixture models were fit to the data to identify trajectory classes. Models with a two-class, three-class, four-class and five-class solution were fitted and compared using AIC, BIC, and the Lo-Mendel-Rubin adjusted likelihood ratio test. Once longitudinal trajectories were identified using growth mixture models, a structural equation model was used to investigate 
whether class membership predicted cognitive outcomes at age 50, after controlling for key covariates. One-step estimation approaches have been criticised in recent years on the basis that including distal outcomes into the measurement model in one step may lead to an unintended and problematic circular relationship in which the classes from the trajectory modelling are determined in part by the distal outcome that they are meant to be predicting. ${ }^{35-37}$ This analysis was therefore conducted in a step-wise fashion to avoid drawbacks associated with one-step estimation methods. Missing data were dealt with using full-information maximum-likelihood estimation (FIML). Analysis was conducted in Mplus V.8. According to Mplus defaults, variances across classes were held equal.

\section{Results}

\section{Missing data and descriptive statistics}

Of the participants who completed the cognitive tests at age $50(n=$ 9385), those with complete information $(n=4625)$ were compared with those with missing data $(n=4760)$. Results revealed that participants with missing data did not differ by gender $(P=0.54)$. However, participants with missing data had significantly lower childhood cognitive scores $(P<0.001)$, higher level of childhood psychological maladjustment $(P<0.001)$, lower level of education $(P<0.001)$, lower socio-economic position in childhood $(P<$ $0.001)$ and adulthood $(P<0.001)$, and more case-level affective symptoms at ages $23(P<0.001), 33(P<0.001), 42 \quad(P<0.001)$ and $50(P<0.001)$.

The imputed data-set was created for the sample of 9385 participants. Sociodemographic information for the participants with complete information for cognitive outcomes, main predictors and covariates ( $n=4625 ; 50.5 \%$ women) and the imputed sample ( $n=9385 ; 50.8 \%$ women) is presented in supplementary Table 3 .

\section{Accumulation of affective symptoms and mid-life cognitive function}

Results from the analysis using a structured modelling approach revealed that the accumulation model was the best fit for the data. For this model, there were no significant differences observed from the saturated model for any of the cognitive outcomes (Table 1 ). This was particularly prominent for memory outcomes (immediate memory: $F=1.81 ; P=0.09$; delayed memory: $F=1.39$, $P=0.22$ ), for which none of the other models (i.e. 'no effect' model, or sensitive period models) fit the data well $(P<0.05$ for all other models).

Fully adjusted regression models revealed significant linear associations, whereby as the number of case-level affective symptoms increased over the life course, mid-life immediate memory $(b=-0.07$, s.e. $=0.03, P=0.01)$, delayed memory $(b=-0.13$, s.e. $=0.04, P<0.001)$ and information processing accuracy $(b=$ 0.18 , s.e. $=0.08, P=0.03$ ) decreased (Table 2). No effects were apparent for verbal fluency or information processing speed in fully adjusted models. Results of the analyses using imputed data were similar (supplementary Tables 4 and 5).

\section{Trajectories of affective symptoms and mid-life cognitive function}

Linear mixed models revealed that a linear trajectory $(\mathrm{AIC}=$ 9020.291, BIC $=9065.883$ ), rather than a quadratic trajectory (AIC $=9021.989, \quad B I C=9075.18)$, fitted adult affective symptom latent variables scores better and were therefore used to identify trajectory classes. When comparing models with a two-class, threeclass, four-class and five-class solution, the five-class solution was found to be the best fit for the data (supplementary Table 6).

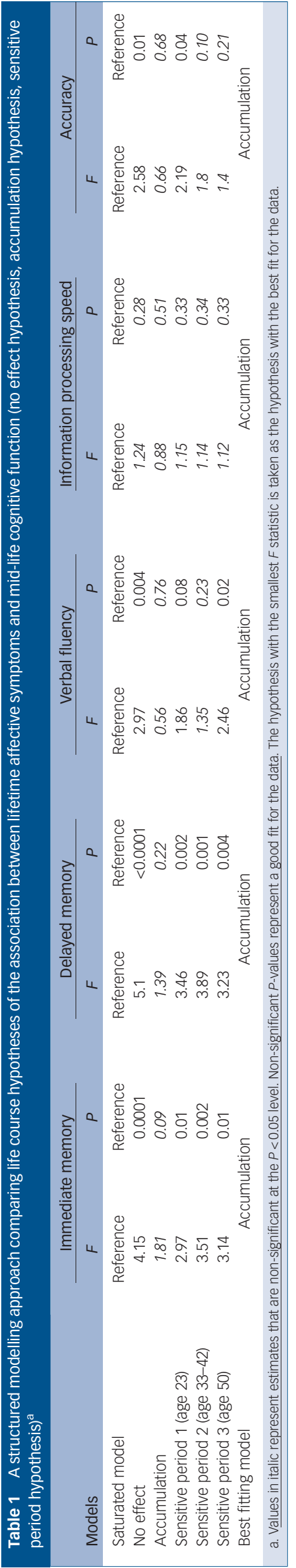




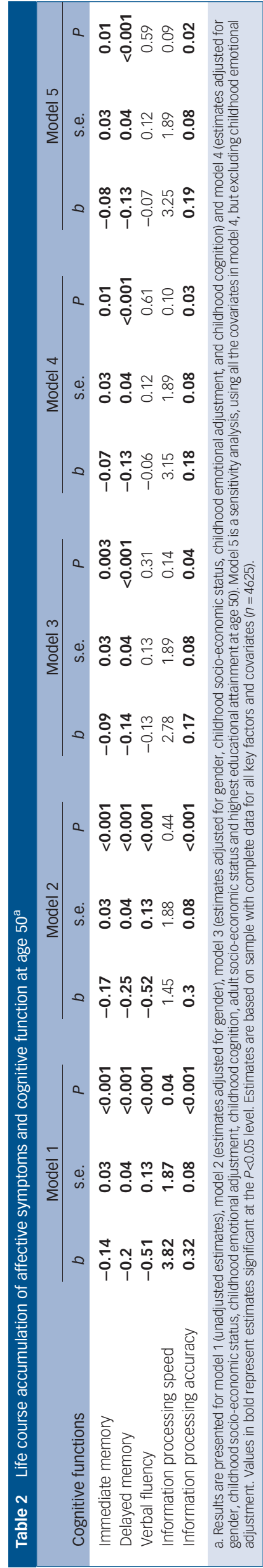

Therefore, five different trajectories of adult affective symptoms were identified (Fig. 1): Class 1 - no affective symptoms (51.4\%); Class 2 - consistent mild/moderate affective symptoms (28.3\%); Class 3 - initially low and increasing to high affective symptoms (5.4\%); Class 4 - initially high and persistently increasing affective symptoms (7.3\%); Class 5 - initially high and decreasing to low affective symptoms (7.5\%).

A fully adjusted structural equation model revealed that class membership predicted cognitive function at age 50 (Table 3). Belonging to a trajectory with initially high and increasing affective symptoms was associated with lower mid-life immediate memory $(b=-0.25$, s.e. $=0.07, P<0.001)$, delayed memory $(b=-0.23$, s.e. $=0.09, P=0.006)$, and verbal fluency scores $(b=-0.79$, s.e. $=$ $0.30, P=0.01$ ), as compared with the trajectory with no affective symptoms. Belonging to a trajectory with initially low and increasing affective symptoms was associated with significantly lower midlife immediate $(b=-0.16$, s.e. $=0.07, P=0.03)$ and delayed memory $(b=-0.23$, s.e. $=0.09, P=0.007)$ and information processing accuracy $(b=0.50$, s.e. $=0.21, P=0.02)$. Belonging to a trajectory with initially high and decreasing affective symptoms was associated with poorer immediate memory $(b=-0.15$, s.e. $=0.07, P=0.04)$. Finally, belonging to a trajectory with consistently mild/moderate affective symptoms was associated with poorer immediate memory $(b=-0.09$, s.e. $=0.04, P=0.03)$. No associations were found for information processing speed and accuracy. Unadjusted and partially adjusted models are reported in supplementary Table 7.

\section{Discussion}

The present study found that accumulation of affective symptoms across three decades of adulthood (from age 23 to age 50) was associated with poorer cognitive function in mid-life: a greater number of case-level affective symptoms was linearly associated with poorer memory and information processing accuracy in mid-life. Analysis of longitudinal trajectories of affective symptoms showed that belonging to a trajectory with a high and increasing level of affective symptoms across adulthood was significantly associated with poorer verbal memory and fluency in mid-life. Belonging to a trajectory with low and increasing affective symptoms was associated with lower verbal memory and information processing accuracy scores at age 50 . Belonging to a trajectory with initially high and decreasing affective symptoms or a trajectory with consistently mild/moderate affective symptoms was associated with lower immediate memory scores in mid-life.

These findings suggest that associations between affective symptoms and cognitive function may be evident even by mid-life, an earlier stage in the life course than considered by many previous studies. ${ }^{38}$ It is now believed that for those experiencing dementia in later life, there is a long preclinical period before cognitive impairment becomes evident. ${ }^{39}$ It is possible that older participants may have already developed cerebral pathology by the time of baseline assessment, even if they are not yet displaying symptoms of cognitive impairment. It is plausible that associations between affective disorders and cognition in older adults may be the result of reverse causality from subtle cognitive changes short of dementia. For this reason, it is important to explore this association earlier in the life course and in a population who have not developed dementia pathology. Mid-life may prove a better age to guarantee forward temporal associations between risk factors, such as affective disorders and subsequent cognitive impairments. ${ }^{40,41}$ Additionally, if associations between affective disorders and cognitive function are already apparent by mid-life, this may be an important window for early intervention. ${ }^{42}$ These results advance previous findings, demonstrating for the first time that effects may be 


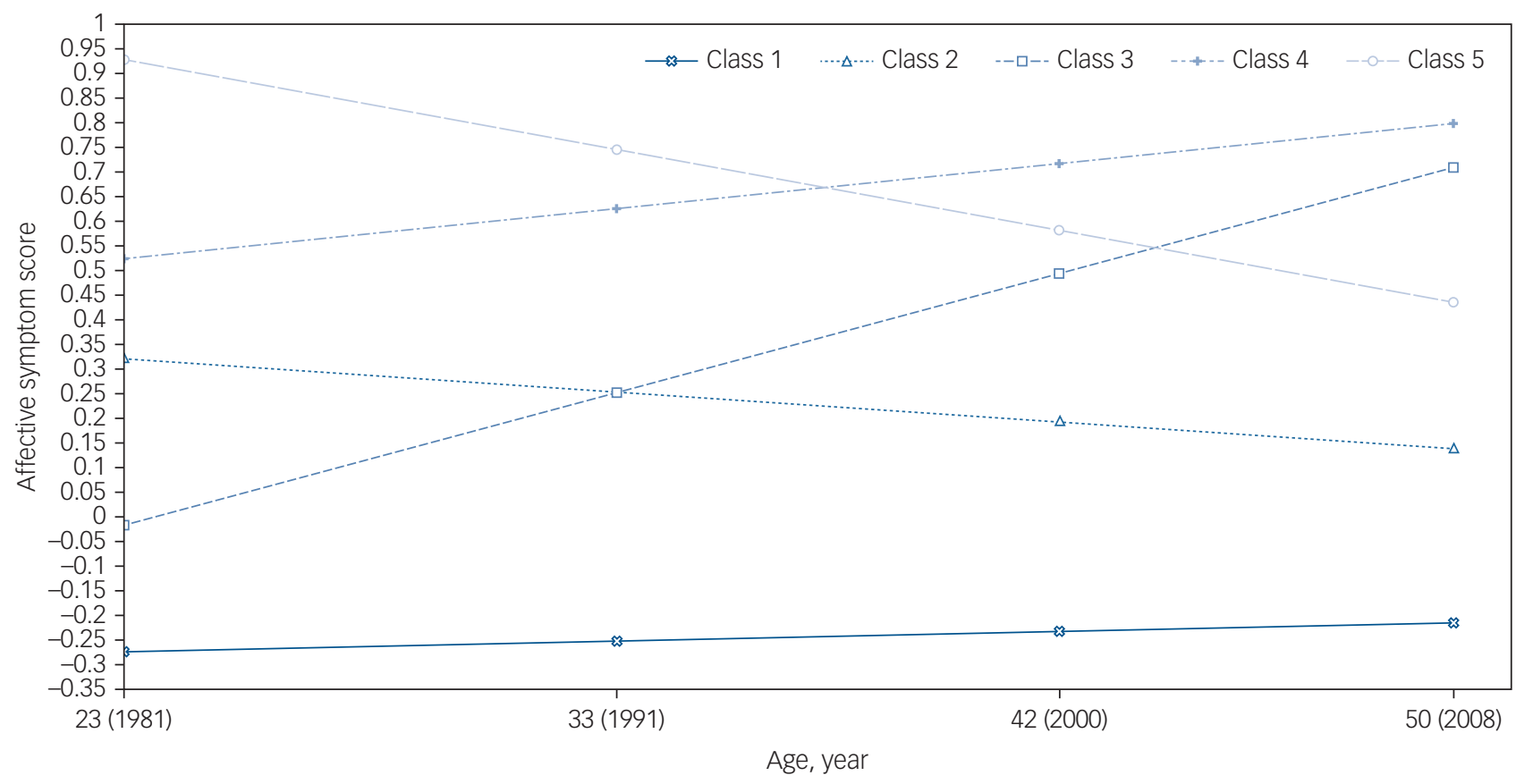

Fig. 1 Longitudinal trajectories of affective symptoms (estimated from five-class growth mixture model).

Class 1: no affective symptoms (51.4\%); class 2: mild/moderate affective symptoms (28.3\%); class 3: initially low and rapidly increasing affective symptoms (5.4\%); class 4: initially high and persistently increasing affective symptoms (7.3\%); class 5: initially high and steadily decreasing affective symptoms (7.5\%).

apparent even many years prior to development of any substantial cognitive deficits and also may be apparent even if no dementia develops. This observation has value in clarifying the temporal order of this association and minimises the issue of reverse causality inherent in studies focusing exclusively on older adults.

These results also suggest that accumulation of multiple, repeated affective episodes can predict poorer cognitive function in mid-life. Although the late-life trajectory of cognitive decline in this current cohort cannot be modelled, the findings add to previous research that reports a monotonic increase in dementia risk with each additional affective episode, by demonstrating that the pattern of accumulating affective episodes is important in predicting mid-life cognitive function in a general population. ${ }^{5}$ Singh-Manoux et al, ${ }^{6}$ investigated depression trajectories over a period of 28 years for a population of individuals who developed dementia up to 2015 (participants aged 65-85 in 2015), and those who remained cognitively healthy, and found an accelerated growth in depressive symptoms during the decade before dementia diagnosis. Our results complement findings from recent studies using data from the MRC National Survey of Health and Development cohort born in 1946, which reported that recurrent case-level affective symptoms were associated with poorer cognitive state (as measured with Addenbrooke's Cognitive Examination third edition), verbal memory, and search speed and accuracy in early old age, ${ }^{8}$ but not with objectively measured cognitive outcomes in late mid-adulthood. ${ }^{7}$ Because prevalence of depression and psychological distress has been increasing in the UK over the past few decades ${ }^{43}$ it is possible that the effect of mental health problems on cognitive ageing can manifest earlier (by mid-life) in younger cohorts, such as individuals in the NCDS born in 1958.

Taken together, these results suggest that verbal memory is affected by the number of affective episodes experienced and by longitudinal trajectories of affective symptoms. Information processing accuracy is affected by the number of affective episodes experienced and by a trajectory of initially low and increasing affective episodes. Verbal fluency scores are affected by a pattern of initially high and increasing accumulation of affective episodes. Strongest effects of affective symptoms were therefore observed on mid-life verbal memory, verbal fluency and information processing accuracy, whereas information processing speed was consistently unaffected by affective symptoms. Several studies have found untreated depressive symptoms persistent over the entire life course may lead to cumulative hippocampal volume loss; ${ }^{44}$ a key structure associated with verbal memory. ${ }^{45}$ This could potentially explain effects of affective problems on memory. It is possible that mid-life may be too early for effects to be observed in information processing speed, and that effects of affective disorders may become apparent on this cognitive domain as individuals transition from mid-life into older adulthood.

\section{Strengths and limitations}

The key strength of the study is a large nationally representative sample with a long follow-up period (three decades). Moreover, multiple cognitive domains were assessed in mid-life, and prospective assessments of affective symptoms using the same instrument from early adulthood to mid-life were available for the analyses. However, results should be interpreted with consideration of a number of limitations. Cognitive function was only assessed at one time point in adulthood (age 50) in the NCDS, and therefore it was not possible to investigate the effects of affective problems on cognitive change over time. Cognitive assessments at this time point were also limited in breadth; single, rather than multiple, cognitive tests were used for each domain (immediate and delayed memory, verbal fluency, information processing speed and accuracy); and functions in other cognitive domains (such as inhibitory processes, attention) known to be affected in people with affective disorders, were not assessed in NCDS. Additionally, although cognitive ability in childhood was controlled for, this does not completely eliminate the possibility of reverse causality, whereby the association may operate in the opposite direction with lower cognitive function leading to higher affective symptoms across the 


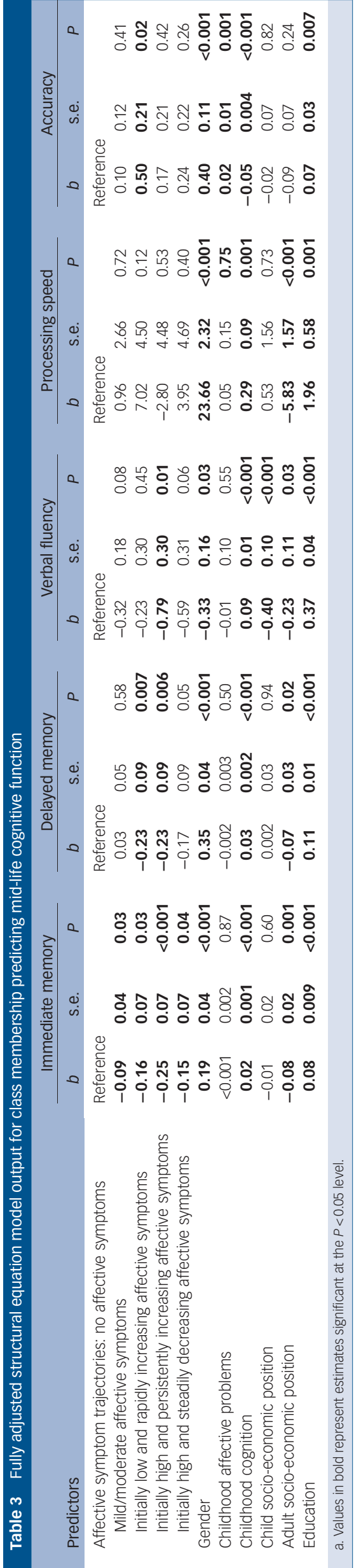

adulthood. ${ }^{46}$ We were also unable to take the effect of medications into account, which may play an important role in this association.

Missing data are inevitable in the long-running cohorts such as NCDS, and indeed there was a lot of missing data that could potentially lead to biased estimates. We have dealt with this by imputing missing data using a multiple imputation approach. The benefits of the multiple imputation approach are that missing data can be dealt with prior to analysis ${ }^{47}$ using a large amount of additional information from other variables available in the data-set. Specifically, this allows extra information that is not included in our main models (such as birth weight and parental education) to be used as auxiliary variables to aid with imputing the missing data. This maximises plausibility of the missing at random assumption. ${ }^{31}$ Notably, the results from imputed data were substantially identical to the ones obtained using a complete data-set and also were consistent and complementary with the analysis using FIML to account for missing data.

\section{Plausible mechanisms}

Both biological and sociobehavioural pathways have been proposed to explain the link between affective disorders and cognitive function. Specifically, hypothalamic-pituitary-adrenal (HPA) axis function has been proposed as one potential mechanism linking affective problems and subsequent cognitive dysfunction. ${ }^{48}$ Affective symptoms and chronic stress may give rise to HPA axis activity and lead to increased glucocorticoid production, which in turn may lead to hippocampal atrophy and cognitive dysfunction. Animal studies exploring response to stress have proposed that conditions of high stress and exogenous glucocorticoids can lead to cognitive impairment. ${ }^{49}$

Another potential pathway involves cardio-metabolic risk factors. Affective problems and psychological distress across the life course have been associated with higher cardio-metabolic risk. $^{50}$ Additionally, cardio-metabolic disorders have also been linked with Alzheimer's disease and cognitive decline. ${ }^{51}$ Related to this, physical health and health behaviours, ${ }^{52}$ including physical activity and exercise may also play an important role in the association between affective problems and cognitive ageing. Beyond this, chronic inflammation plays a role in both depression and dementia, and as such may act as an important pathway between the two. Moreover, $\beta$-amyloid deposition is known to play an important role in the pathogenesis of dementia ${ }^{53}$ and has additionally been associated with major depression. ${ }^{54}$ Educational attainment ${ }^{55}$ and socio-economic position ${ }^{23}$ may also play a role in the association between affective problems and cognitive ageing. It is plausible that the association between affective problems and cognitive ageing is underpinned by a complex interaction of biological and sociobehavioural mechanisms, rather than by one single aetiological determinant. $^{2}$

\section{Implications}

In conclusion, the present study suggests that individuals with affective symptoms in adulthood are at increased risk of poorer cognitive outcomes by mid-life. This finding has implications for prevention efforts, as the asymptomatic phase before development of dementia pathology may be a critical window to target for early intervention. ${ }^{42}$ Additionally, in the absence of pathological change, one further important avenue of research is to investigate whether effective treatment and management of affective problems early in life can reduce risk of poor cognitive outcomes and promote healthy cognitive ageing. Future research should also focus on determining the biological and sociobehavioural mechanisms that underpin the association between affective and cognitive factors. All interventions to promote and sustain healthy ageing are important to health policy development in an ageing population. 
Amber John, MA, PhD Student, EDGE Lab, School of Psychology, University of Sussex UK; Sarah-Naomi James, PhD, Postdoctoral researcher, MRC Unit for Lifelong Health and Ageing at UCL, UK; Urvisha Patel, MSC, MSc Student, EDGE Lab, School of Psychology, University of Sussex, UK; Jennifer Rusted, PhD, Professor of Experimenta Psychology, School of Psychology, University of Sussex, UK; Marcus Richards, PhD, Programme Leader. MRC Unit for Lifelong Health and Ageing at UCL, UK; Darya Gaysina PhD, Senior Lecturer in Psychology, EDGE Lab, School of Psychology, University of Sussex, UK

Correspondence: Darya Gaysina, EDGE Lab, School of Psychology, University of Sussex Pevensey 1 Building, Brighton BN1 9QH, UK. Email: d.gaysina@sussex.ac.uk

First received 23 Aug 2018, final revision 11 Jan 2019, accepted 11 Jan 2019

\section{Funding}

We would like to acknowledge the Economic and Social Research Council (ESRC) for supporting this project (Grant number: ES/J500173/1).

\section{Acknowledgements}

We would like to acknowledge the Economic and Social Research Council (ESRC) for supporting this project. We would also like to thank the Centre for Longitudinal Studies (CLS), UCL Institute of Education for use of the data. We also acknowledge the UK Data Service for making data freely available. Finally, we would like to thank the cohort members who have dedicated their time to the National Child Development Study.

\section{Supplementary material}

Supplementary material is available online at https://doi.org/10.1192/bjp.2019.24.

\section{References}

1 John A, Patel U, Rusted J, Richards M, Gaysina D. Affective problems and decline in cognitive state in older adults: a systematic review and meta-analysis. Psychol Med 2018; 49(3): 353-65.

2 Da Silva J, Gonçalves-Pereira M, Xavier M, Mukaetova-Ladinska EB. Affective disorders and risk of developing dementia: systematic review. Br J Psychiatry 2013; 202: 177-86.

3 Gulpers B, Ramakers I, Hamel R, Köhler S, Oude Voshaar R, Verhey F. Anxiety as a predictor for cognitive decline and dementia: a systematic review and metaanalysis. Am J Geriatr Psychiatry 2016; 24: 823-42.

4 Singh-Manoux A, Akbaraly TN, Marmot M, Melchior M, Ankri J, Sabia S, et al. Persistent depressive symptoms and cognitive function in late midlife: the Whitehall II study. J Clin Psychiatry 2010; 71: 1379.

5 Kessing LV, Andersen PK. Does the risk of developing dementia increase with the number of episodes in patients with depressive disorder and in patients with bipolar disorder? J Neurol Neurosurg Psychiatry 2004; 75: 1662-6.

6 Singh-Manoux A, Dugravot A, Fournier A, Abell J, Ebmeier K, Kivimäki M, et al. Trajectories of depressive symptoms before diagnosis of dementia. JAMA Psychiatry 2017; 74: 712.

7 Richards M, Barnett JH, Xu MK, Croudace TJ, Gaysina D, Kuh D, et al. Lifetime affect and midlife cognitive function: prospective birth cohort study. $\mathrm{Br} J$ Psychiatry 2014; 204: 194-9.

8 James S-N, Davis D, O'Hare C, Sharma N, John A, Gaysina D, et al. Lifetime affective problems and later-life cognitive state: over 50 years of follow-up in a British Birth Cohort Study. J Affect Disord 2018; 241: 348-355.

9 Power C, Elliott J. Cohort profile: 1958 British birth cohort (National Child Development Study). Int J Epidemiol 2006; 35: 34-41.

10 University of London, Institute of Education, Centre for Longitudinal Studies. National Child Development Study: Childhood Data, Sweeps 0-3, 1958-1974. [data collection] 3rd Edition: SN 5565. National Birthday Trust Fund, National Children's Bureau [original data producer(s)]. National Birthday Trust Fund 2014. http://doi.org/10.5255/UKDA-SN-5565-2

11 University of London, Institute of Education, Centre for Longitudinal Studies. National Child Development Study: Sweep 4, 1981, and Public Examination Results, 1978. [data collection] 2nd Edition: SN 5566. National Children's Bureau [original data producer(s)]. National Children's Bureau, 2008. http://doi. org/10.5255/UKDA-SN-5566-1

12 University of London, Institute of Education, Centre for Longitudinal Studies. National Child Development Study: Sweep 5, 1991. [data collection] 2nd Edition: SN 5567. City University, Social Statistics Research Unit [original data producer(s)]. City University, Social Statistics Research Unit, 2008. http://doi. org/10.5255/UKDA-SN-5567-1

13 University of London, Institute of Education, Centre for Longitudinal Studies. National Child Development Study: Sweep 6, 1999-2000. [data collection] 2nd Edition: SN 5578. Joint Centre for Longitudinal Research [original data producer(s)]. Joint Centre for Longitudinal Research, 2008. http://doi.org/10.5255/UKDA-SN5578-12008

14 University of London, Institute of Education, Centre for Longitudinal Studies. National Child Development Study: Sweep 8, 2008-2009. [data collection] 3rd Edition: SN 6137. UK Data Service, 2012. http://doi.org/10.5255/UKDA-SN6137-2

15 Brown M, Dodgeon B. NCDS Cognitive Assessments at age 50: Initial Results. CLS Cohort Studies, Working Paper. Centre for Longitudinal Studies, Institute of Education, University of London, 2010.

16 Hope S, Power C, Rodgers B. Does financial hardship account for elevated psychological distress in lone mothers? Soc Sci Med 1999; 49: 1637-49.

17 Sacker A, Cable N. Do adolescent leisure-time physical activities foster health and well-being in adulthood? Evidence from two British birth cohorts. Eur J Public Health 2006; 16: 331-5.

18 Lacey R, Bartley M, Pikhart H, Stafford M, Cable N, Coleman L. Parental separation and adult psychological distress: evidence for the "reduced effect" hypothesis? Longit Life Course Stud 2012; 3: 359-68.

19 Bowling A, Pikhartova J, Dodgeon B. Is mid-life social participation associated with cognitive function at age 50 ? Results from the British National Child Development Study (NCDS). BMC Psychol 2016; 4: 58.

20 MCGurn B, Deary IJ, Starr JM. Childhood cognitive ability and risk of late-onset Alzheimer and vascular dementia. Neurology 2008; 71: 1051-6.

21 Lupien SJ, McEwen BS, Gunnar MR, Heim C. Effects of stress throughout the lifespan on the brain, behaviour and cognition. Nat Rev Neurosci 2009; 10 : 434-45.

22 Kaplan GA, Turrell G, Lynch JW, Everson SA, Helkala E-L, Salonen JT. Childhood socioeconomic position and cognitive function in adulthood. Int J Epidemiol 2001; 30: 256-63.

23 Sattler $C$, Toro P, Schönknecht P, Schröder J. Cognitive activity, education and socioeconomic status as preventive factors for mild cognitive impairment and Alzheimer's disease. Psychiatry Res 2012; 196: 90-5.

24 Brayne C, Ince PG, Keage HAD, McKeith IG, Matthews FE, Polvikoski T, et al. Education, the brain and dementia: neuroprotection or compensation? Brain 2010; 133: 2210-6

25 Mcdermott PA, Watkins MW. Dimensions of maladaptive behavior among kindergarten level children. Counc Except Child Dimens 2017; 7: 11-7.

26 Elliott J, Lawrence J. Refining Childhood Social Class Measures in the 1958 British Cohort Study in the 1958 British Birth Cohort Study. University of London: Centre for Longitudinal Studies, Institute of Education, 2014.

27 Mishra G, Nitsch D, Black S, De Stavola B, Kuh D, Hardy R. A structured approach to modelling the effects of binary exposure variables over the life course. Int J Epidemiol 2009; 38: 528-37.

28 Murray ET, Mishra GD, Kuh D, Guralnik J, Black S, Hardy R. Life course models of socioeconomic position and cardiovascular risk factors: 1946 birth cohort. Ann Epidemiol 2011; 21: 589-97.

29 van Buuren S, Groothuis-Oudshoorn K. mice : multivariate imputation by chained equations in R. J Stat Softw 2011; 45: 1-68.

30 Azur MJ, Stuart EA, Frangakis C, Leaf PJ. Multiple imputation by chained equations: what is it and how does it work? Int J Methods Psychiatr Res 2011; 20: 40-9.

31 Coley N, Gardette V, Cantet C, Gillette-Guyonnet S, Nourhashemi F, Vellas B, et al. How should we deal with missing data in clinical trials involving Alzheimer's disease patients? Curr Alzheimer Res 2011; 8: 421-33.

32 Solís C B, Kelly-Irving M, Fantin R, Darnaudéry M, Torrisani J, Lang T, et al. Adverse childhood experiences and physiological wear-and-tear in midlife: findings from the 1958 British birth cohort. Proc Natl Acad Sci 2015; 112: E738-46.

33 Kelly-irving M, Lepage B, Dedieu D, Lacey R, Cable N, Bartley M, et al. Childhood adversity as a risk for cancer : findings from the 1958 British birth cohort study. BMC Public Health 2013; 13: 767.

34 Blane $D$, Wahrendorf $M$, Webb E, Netuveli G. Life course influences on quality of life at age 50 years: evidence from the National Child Development Study (1958 British birth cohort study). Longit Life Course Stud 2012; 3: 346-58.

35 Vermunt JK. Latent class modeling with covariates: two improved three-step approaches. Polit Anal 2010; 18: 450-69.

36 Bakk Z, Vermunt JK. Robustness of stepwise latent class modeling with continuous distal outcomes. Struct Equ Model A Multidiscip J 2015; 23: 20-31.

37 Zhu Y, Steele F, Moustaki I. A general 3-step maximum likelihood approach to estimate the effects of multiple latent categorical variables on a distal outcome. Struct Equ Model A Multidiscip J 2017; 24: 643-56. 
38 Ganguli M, Du Y, Dodge HH, Ratcliff GG, Chang C-CH. Depressive symptoms and cognitive decline in late life. Arch Gen Psychiatry 2006; 63: 153.

39 Morris JC. Early-stage and preclinical Alzheimer disease. Alzheimer Dis Assoc Disord 2005; 19: 163

40 Lancaster C, Tabet N, Rusted J. The elusive nature of APOE $\varepsilon 4$ in mid-adulthood: understanding the cognitive profile. J Int Neuropsychol Soc 2017; 23: 239-53.

41 Exalto LG, Quesenberry CP, Barnes D, Kivipelto M, Biessels GJ, Whitmer RA. Midlife risk score for the prediction of dementia four decades later. Alzheimer's Dement 2014; 10: 562-70.

42 sperling RA, Jack CR, Aisen PS. Testing the right target and right drug at the right stage. Sci Transl Med 2011; 3: 111cm33.

43 Fink E, Patalay P, Sharpe H, Holley S, Deighton J, Wolpert M. Mental health difficulties in early adolescence: a comparison of two cross-sectional studies in England from 2009 to 2014. J Adolesc Heal 2015; 56: 502-7.

44 Sheline $\mathrm{Yl}$, Gado $\mathrm{MH}$, Kraemer HC. Untreated depression and hippocampal volume loss. Am J Psychiatry 2003; 160: 1516-8.

45 Bird CM, Burgess N. The hippocampus and memory: insights from spatial processing. Nat Rev Neurosci 2008; 9: 182-94.

46 Hatch SL, Jones PB, Kuh D, Hardy R, Wadsworth MEJ, Richards M. Childhood cognitive ability and adult mental health in the British 1946 birth cohort. Soc SCi Med 2007; 64: 2285-96.

47 Schafer JL, Graham JW. Missing data : our view of the state of the art. Psychol Methods 2002; 7(2): 147-77.

48 Byers A, Yaffe K. Depression and risk of developing dementia. Nat Rev Neurol 2011; 7: 323-31.
49 Park CR, Zoladz PR, Conrad CD, Fleshner M, Diamond DM. Acute predator stress impairs the consolidation and retrieval of hippocampus-dependent memory in male and female rats. Learn Mem 2008; 15: 271-80.

50 Winning A, Glymour MM, McCormick MC, Gilsanz P, Kubzansky LD. Psychological distress across the life course and cardiometabolic risk findings from the 1958 British birth cohort study. J Am Coll Cardiol 2015; 66 1577-86.

51 Bhat NR. Linking cardiometabolic disorders to sporadic Alzheimer's disease: a perspective on potential mechanisms and mediators. J Neurochem 2010; 115 $551-62$

52 Ströhle A. Physical activity, exercise, depression and anxiety disorders. J Neural Transm 2009; 116: 777-84.

53 Hardy J, Selkoe DJ. The amyloid hypothesis of Alzheimer's disease: progress and problems on the road to therapeutics. Science 2002; 297: 353-6.

54 Wu KY, Hsiao IT, Chen CS, Chen $\mathrm{CH}$, Hsieh CJ, Wai YY, et al. Increased brain amyloid deposition in patients with a lifetime history of major depression: evidenced on 18F-florbetapir (AV-45/Amyvid) positron emission tomography. Eur J Nucl Med Mol Imaging 2014; 41: 714-22.

55 Geerlings MI, Schmand B, Braam AW, Jonker IC. Depressive symptoms and risk of Alzheimer's disease in more highly educated older people. J Am Geriatr Soc 2000; 48: 1092-7. 